\title{
Radiometric Measurement Comparisons Using Transfer Radiometers in Support of the Calibration of NASA's Earth Observing System (EOS) Sensors
}

\author{
James J. Butler ${ }^{\mathbf{a}}$, B. Carol Johnson ${ }^{\mathrm{b}}$, Steven W. Brown ${ }^{\mathrm{b}}$, Howard W. Yoon ${ }^{\mathrm{b}}$, Robert A. Barnes ${ }^{\mathrm{c}}$, Brian L. \\ Markham ${ }^{\mathrm{d}}$, Stuart F. Biggare, Edward F. Zalewskie, Paul R. Spyak ${ }^{e}$, John W. Cooper ${ }^{\mathrm{f}}$, Fumihiro \\ Sakuma ${ }^{\mathrm{g}}$ \\ ${ }^{2}$ NASA Goddard Space Flight Center, Code 920.1, Greenbelt, MD 20771 \\ bational Institute of Science and Technology, Optical Technology Division, Gaithersburg, MD 20899

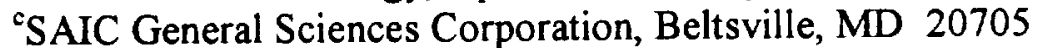 \\ NASA Goddard Space Flight Center, Code 923, Greenbelt, MD 20771 \\ 'University of Arizona, Optical Sciences Center, Remote Sensing Group, Tucson, AZ 85721 \\ ${ }^{f}$ Raytheon STX, Lanham, MD 20706 \\ BNational Research Laboratory of Metrology, Thermophysical Metrology Department, \\ 1-1-4, Umezono, Tsukuba-Shi, Ibaraki, 302 Japan
}

\begin{abstract}
EOS satellite instruments operating in the visible through the shortwave infrared wavelength regions (from $0.4 \mu \mathrm{m}$ to $2.5 \mu \mathrm{m}$ ) are calibrated prior to flight for radiance response using integrating spheres at a number of instrument builder facilities. The traceability of the radiance produced by these spheres with respect to international standards is the responsibility of the instrument builder, and different calibration techniques are employed by those builders. The National Aeronautics and Space Administration's (NASA's) Earth Observing System (EOS) Project Science Office, realizing the importance of preflight calibration and cross-calibration, has sponsored a number of radiometric measurement comparisons, the main purpose of which is to validate the radiometric scale assigned to the integrating spheres by the instrument builders. This paper describes the radiometric measurement comparisons, the use of stable transfer radiometers to perform the measurements, and the measurement approaches and protocols used to validate integrating sphere radiances. Stable transfer radiometers from the National Institute of Standards and Technology, the University of Arizona Optical Sciences Center Remote Sensing Group, NASA's Goddard Space Flight Center, and the National Research Laboratory of Metrology in Japan, have participated in these comparisons. The approaches used in the comparisons include the measurement of multiple integrating sphere lamp levels, repeat measurements of select lamp levels, the use of the stable radiometers as external sphere monitors, and the rapid reporting of measurement results. Results from several comparisons are presented. The absolute radiometric calibration standard uncertainties required by the EOS satellite instruments are typically in the $\pm 3 \%$ to $\pm 5 \%$ range. Preliminary results reported during eleven radiometric measurement comparisons held between February 1995 and May 1998 have shown the radiance of integrating spheres agreed to within $\pm 2.5 \%$ from the average at blue wavelengths and to within $\pm 1.7 \%$ from the average at red and near infrared wavelengths. This level of agreement lends confidence in the use of the transfer radiometers in validating the radiance scales assigned by EOS instrument calibration facilities to their integrating sphere sources.
\end{abstract}

Keywords: calibration, integrating spheres, radiance, transfer radiometers

For further author information -

J.J.B. (correspondence) email: butler@ltpmail.gsfc.nasa.gov; telephone 301-614 5942; facsimile 301-614-5970. 


\section{INTRODUCTION}

The international Earth remote sensing scientific community has unanimously recognized the need for long tcrm precise and accurate remote sensing data to assess natural and anthropogenic effects on Earth's climate and environment. In an effort to provide those data, NASA's EOS was implemented. It is an 18 year, international, multi-satellite, multi-instrument program in global remote sensing of the Earth. The goal of the EOS mission is to advance the scientific understanding of the Earth system and its changes on a global scale through a deeper understanding of the components of that system and their many interactions. The precisions and accuracies of the EOS instruments' measurements are directly derived from the science requirements of the remote sensing community, the ultimate users and interpreters of EOS data. These precision and accuracy requirements are state-of-the art for measurements made by on-orbit satellite instruments and, in some cases, even for measurements made by instruments in the more hospitable environment of the laboratory.

In order to achieve required EOS instrument precision and accuracy requirements and thereby correctly interpret long term EOS data, on-orbit changes in the satellite instruments must be differentiated from actual changes in the remotely sensed Earth scenes. The ability to differentiate these processes initially depends on careful prelaunch calibration of the satellite instruments with respect to physical standards and thorough prelaunch characterization of the instruments at the system and subsystem levels. Following launch, the ability to differentiate these processes depends on the ability of instrument on-board calibration systems to monitor changes to the prelaunch calibration and on comparisons of satellite instrument measurements with measurements made by calibrated ground-based, airborne, and other satellite borne instruments. The multi-instrument nature of the EOS program underscores the need to relate satellite, ground-based, and airborne instrument measurements through a common set of physical standards and processes. It is only through this process that biases between instrument data can be eliminated and data from a number of instruments can be used with confidence by Earth remote sensing scientists.

The pre-launch calibrations of EOS satellite, ground-based, and airborne instruments operating in the visible through shortwave infrared wavelength regions (i.e. $0.4 \mu \mathrm{m}$ to $2.5 \mu \mathrm{m}$ ) are placed on a common scale through the use of available physical standard sources and artifacts provided by national standards laboratories. The validation of the calibration of these EOS instruments is accomplished using ultra-stable transfer radiometers designed, built, and independently calibrated by national standards laboratories and secondary standards calibration facilities. Transfer radiometers from the National Institute of Standards and Technology (NIST), the National Research Laboratory of Metrology-National Space Development Agency of Japan-Japan Resources Observation System Organization (NRLM-NASDA-JAROS), the University of Arizona (UA) Optical Sciences Center Remote Sensing Group, and NASA's Goddard Space Flight Center (GSFC) have participated in eleven radiometric measurement comparisons at a number of EOS instrument calibration facilities in the United States and Japan. This paper describes the transfer radiometers, outlines the comparison goals, and presents preliminary measurement results.

\section{THE TRANSFER RADIOMETERS}

The transfer radiometers that have participated in the radiometric measurement comparisons are briefly described below. The wavelengths and bandwidths of the filter transfer radiometers are provided in Table 1.

\subsection{NIST TRANSFER RADIOMETERS}

NIST has participated in EOS radiometric comparisons with the NISTVisible/near infrared Transfer Radiometer (VXR), the Sea-viewing Wide Field-of-view Sensor (SeaWiFS) Transfer Radiometer (SXR), and the NIST Shortwave Infrared Transfer Radiometer (SWIXR). The NIST VXR and SXR are filter radiometers of similar optical design'. The VXR and SWIXR were built by NIST for the EOS Project Science Office, while the SXR was built for the SeaWiFS Project Office. Beginning in May 1998, the NIST Shortwave Infrared Transfer Radiometer (SWIXR) participated in radiometric measurement comparisons ${ }^{2}$. The SWIXR is a double monochromator-based scanning spectroradiometer incorporating all-reflective input optics and a liquid nitrogen cooled indium antimonide (InSb) detector. The operating wavelength range of the SWIXR is 0.8 $\mu \mathrm{m}$ to $2.5 \mu \mathrm{m}$. 


\subsection{UA TRANSFER RADIOMETERS}

The UA Visible/Near Infrared radiometer (UA VNIR) ${ }^{3}$ and the UA Shortwave Infrared transfer radiometer (UA SWIR) have participated in EOS radiometric measurement comparisons. The UA VNIR is a filter radiometer with seven channels corresponding to spectral bands in the Moderate Resolution Imaging Spectroradiometer (MODIS), the Advanced Spaceborne Thermal Emission and Reflection Radiometer (ASTER), and the Multi-angle Imaging SpectroRadiometer (MISR) EOS instruments. The UA SWIR is a nine channel filter radiometer employing a liquid nitrogen cooled InSb detector. The SWIR channels are chosen to correspond with 4 MODIS and 5 ASTER bands.

\begin{tabular}{|c|c|c|c|c|c|c|c|}
\hline \multicolumn{4}{|c|}{ NIST } & \multicolumn{3}{c|}{ NRLM/NASDA/JAROS } \\
\hline \multicolumn{2}{|c|}{ VXR } & \multicolumn{2}{c|}{ SXR } & \multicolumn{2}{c|}{ VNIR } & \multicolumn{2}{c|}{ SWIR } \\
\hline $\begin{array}{c}\text { Wave- } \\
\text { length } \\
\text { (nm) }\end{array}$ & $\begin{array}{c}\text { Band- } \\
\text { width } \\
\text { (nm) }\end{array}$ & $\begin{array}{c}\text { Wave- } \\
\text { length } \\
(\mathrm{nm})\end{array}$ & $\begin{array}{c}\text { Band- } \\
\text { width } \\
(\mathrm{nm})\end{array}$ & $\begin{array}{c}\text { Wave- } \\
\text { length } \\
(\mathrm{nm})\end{array}$ & $\begin{array}{c}\text { Band- } \\
\text { width } \\
\text { (nm) }\end{array}$ & $\begin{array}{c}\text { Wave- } \\
\text { length } \\
\text { (nm) }\end{array}$ & $\begin{array}{c}\text { Band- } \\
\text { width } \\
\text { (nm) }\end{array}$ \\
\hline 411.8 & 10.8 & 411.5 & 10.8 & 445 & 19 & 1608 & 150 \\
\hline 441.0 & 10.6 & 441.6 & 10.3 & 490 & 20 & 2181 & 170 \\
\hline 548.4 & 10.4 & 487.1 & 10.6 & 567 & 24 & & \\
\hline 661.4 & 9.6 & 548.0 & 10.4 & 561 & 48 & & \\
\hline 775.5 & 11.6 & 661.8 & 9.57 & 650 & 56 & & \\
\hline 870.0 & 10 & 774.8 & 11.6 & 670 & 18 & & \\
\hline & & & & 764 & 37 & & \\
\hline & & & & 807 & 72 & & \\
\hline & & & & 864 & 42 & & \\
\hline
\end{tabular}

\begin{tabular}{|c|c|c|c|c|c|}
\hline \multicolumn{4}{|c|}{ UA } & \multicolumn{2}{c|}{ NASA's GSFC } \\
\hline \multicolumn{2}{|c|}{ VNIR } & \multicolumn{2}{c|}{ SWIR } & \multicolumn{2}{c|}{ LXR } \\
\hline $\begin{array}{c}\text { Wave- } \\
\text { length } \\
\text { (nm) }\end{array}$ & $\begin{array}{c}\text { Band- } \\
\text { width } \\
\text { (nm) }\end{array}$ & $\begin{array}{c}\text { Wave- } \\
\text { length } \\
\text { (nm) }\end{array}$ & $\begin{array}{c}\text { Band- } \\
\text { width } \\
\text { (nm) }\end{array}$ & $\begin{array}{c}\text { Wave- } \\
\text { length } \\
\text { (nm) }\end{array}$ & $\begin{array}{c}\text { Band- } \\
\text { width } \\
\text { (nm) }\end{array}$ \\
\hline 412.8 & 14.9 & 1243.5 & 15.7 & 440 & 11 \\
\hline 441.8 & 11.8 & 1380.8 & 29.0 & 481 & 61 \\
\hline 488.0 & 9.6 & 1646.0 & 23.4 & 561 & 76 \\
\hline 550.3 & 9.9 & 2133.6 & 55.1 & 661 & 9 \\
\hline 666.6 & 9.8 & 2164.3 & 40.8 & 662 & 60 \\
\hline 746.9 & 10.6 & 2207.9 & 44.5 & 827 & 110 \\
\hline 868.1 & 14.0 & 2263.0 & 49.3 & & \\
\hline & & 2332.3 & 63.1 & & \\
\hline & & 2403.2 & 70.3 & & \\
\hline
\end{tabular}

Note: The NIST, UA VNIR, and NASA's GSFC wavelengths and bandwidths are the result of moment analyses. The UA SWIR center wavelengths are the result of a moment analysis, and the bandwidths are the result of a full-width-at halfmaximum analysis. The NRLM-NASDA-JAROS wavelengths are center values calculated from the $50 \%$ response points and the bandwidths are full-width at half-maximum values.

Table 1. Wavelengths and Bandwidths of the Filter Transfer Radiometers 


\subsection{NASA's GSFC LANDSAT TRANSFER RADIOMETER}

The NASA's GSFC Landsat Transfer Radiometer (LXR) ${ }^{4}$ participated in a number of EOS radiometric measurement comparisons. The LXR is a filter radiometer of similar optical design to the SXR and VXR; it was built by Reyer Corporation for the Landsat Enhanced Thematic Mapper Plus (ETM) Science Office.

\subsection{NRLM-NASDA-JAROS TRANSFER RADIOMETERS}

The NRLM-NASDA transfer radiometers include six, single fixed wavelength filter radiometers operating in the visible through near infrared region ${ }^{5}$. The NRLM-JAROS transfer radiometers include three, single fixed wavelength filter radiometers operating in the visible through near infrared region and two, single fixed wavelength filter radiometers operating the shortwave infrared region ${ }^{6}$. The center wavelengths and bandwidths of the NRLM-NASDA and NRLM-JAROS radiometer filters were selected to closely match bands in the Ocean Color and Temperature Scanner (OCTS) and ASTER satellite instruments, respectively.

\subsection{NASA's GSFC 746/INTEGRATING SPHERE IRRADIANCE COLLECTOR}

The NASA's GSFC 746/Integrating Sphere Irradiance Collector (ISIC) is a scanning single-grating monochromator-based spectro-radiometer equipped with a $10.2 \mathrm{~cm}$ diameter collection sphere at the monochromator entrance port ${ }^{7}$. Using silicon photodiode and lead sulfide detectors, the 746/SIC transfers the irradiance calibration from a NIST calibrated irradiance standard lamp to the integrating sphere being measured. A knowledge of the aperture areas of the sphere under test and the ISIC collection sphere coupled with a knowledge of the distance between the two apertures, enables the radiance of the sphere under test to be calculated. In the visible/near infrared wavelength region from $400 \mathrm{~nm}$ to $1000 \mathrm{~nm}$, the $746 / I S I C$ obtains data in $10 \mathrm{~nm}$ steps with a bandwidth of $10 \mathrm{~nm}$. In the shortwave infrared wavelength region from $1000 \mathrm{~nm}$ to $1600 \mathrm{~nm}$, data is obtained every $20 \mathrm{~nm}$ with a bandwidth of $20 \mathrm{~nm}$. In the wavelength region from $1600 \mathrm{~nm}$ to $2400 \mathrm{~nm}$, data is obtained every $20 \mathrm{~nm}$ with a bandwidth of $40 \mathrm{~nm}$.

\section{THE RADIOMETRIC MEASUREMENT COMPARISONS}

Planning for a radiometric measurement comparison begins with the determination of exact dates for the comparison to be held at the host institution. The host institution for the comparison is the facility where the satellite, ground-based, or airborne instrument is calibrated using the calibration source to be measured. The exact dates for the comparison are determined through the consensus agreement of the host institution and the institutions which plan to participate with their transfer radiometers. Following the determination of the comparison dates and participants, the EOS Calibration Scientist, as a member of the EOS Project Science Office, drafts a measurement plan and circulates the plan among the participating institutions. The measurement plan is designed to address a number of issues concerning the calibration and operation of the integrating sphere to be measured. These issues include the following:

1. Validation of the calibration of the integrating sphere over a range of output radiances similar to those realized while using the sphere to calibrate satellite, ground-based, or airborne instruments;

2. Measurement of the stability of the radiance from the integrating sphere using transfer radiometers as sphere output monitors;

3. Measurement of the repeatability of the radiance from the integrating sphere through repeated measurements of selected sphere levels;

4. Examination of the overall operation, measurement procedures, and documentation of the operation of the integrating sphere by the host institution.

Item 1, the validation of the radiometric scale assigned to the integrating sphere by the host institution against the radiance scale maintained by national standards laboratories, is the primary goal of the radiometric measurement comparison. The ${ }^{1}$ ability to realize this primary goal is dependent on the measurement of items 2 and 3 , the sphere stability and repeatability,

\footnotetext{
- Certain commercial equipment, instruments, or materials are identified in this technical memorandum to foster understanding. Such indentification does not imply recommendation or endorsement by NIST, nor does it imply that the materials or equipment identified are necessarily the best available for the purpose.
} 
respectively. A secondary goal of the radiometric measurement comparison is to make simultaneous, direct comparisons of measurements made by the host institution, national standards laboratories, and other participating institutions involved in the calibration of ground-based and airborne remote sensing instrumentation. A tertiary goal is given in item 4, that is, to evaluate the host institutions measurement procedures and results in terms of basic metrology and good measurement practice. In order to achieve these goals, the host institution must either participate in the measurement comparison or provide recently measured radiance data on their integrating sphere to the measurement participants.

Upon agreement by the host and participating institutions on the measurement plan, the participants arrive at the host institution on the day designated in the plan, unpack, setup, and warmup their equipment. The equipment remains powered, night and day, for the duration of the comparison. Following equipment warmup, the host and participating institutions hold a meeting to discuss and make final refinements to the measurement plan and to distribute comparison log sheets. Actual measurements on the integrating sphere under test begin the following morning. The participants first synchronize their data acquisition system clocks to a common time. During the measurements, each participating institution records the time of their measurement, ancillary measurement information, and the data filenames on the log sheets. The host institution is responsible for operating the integrating sphere under test, recording the sphere lamp currents/voltages, and recording and archiving sphere monitor detector readings. At the end of each measurement day, a meeting is scheduled at which each participating institution reports its measured radiances. This "rapid results" meeting gives a quick and very preliminary indication of the overall agreement or disagreement of the data. Significant discrepancies between results presented at this meeting can be used to indicate areas which should be examined in advance of the next day's measurements. At the end of the comparison, each participant provides the EOS Calibration Scientist an electronic copy of their data files. The host institution provides a copy of their record of sphere lamp currents and voltages and data obtained from sphere monitor detectors.

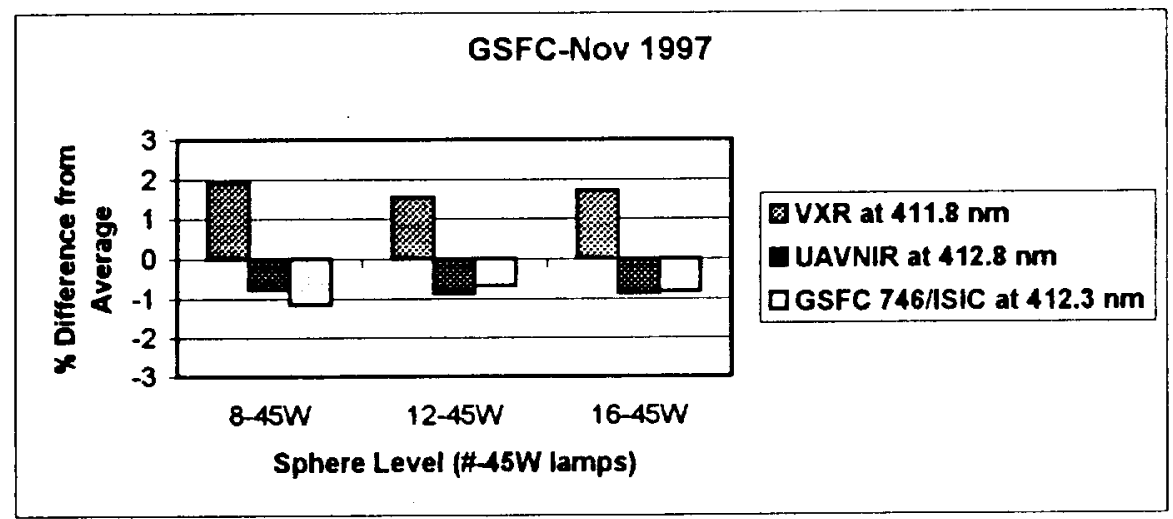

Figure 1. Percent difference of $412 \mathrm{~nm}$ radiance measurements made by the NIST VXR, the University of Arizona VNIR, and the GSFC 746/ISIC radiometers from the average. These measurements were performed at GSFC in November 1997.

From February 1995 to the present, eleven EOS radiometric measurement comparisons have been held. The dates, locations, host institution, participating institutions and transfer radiometers, and integrating sphere or spheres measured are presented in Table 2.

\section{PRELIMINARY RADIOMETRIC MEASUREMENT COMPARISON RESULTS}

The measurement results presented in this paper, restricted to the visible/near infrared, are those reported by the radiometric comparison participants at the end of each comparison day. As such, these results are viewed by the comparison participants to be preliminary. These data are produced by each participating group using their own calculational techniques. An improvement in the agreement of these data is achieved through the implementation of common algorithms in the analysis of the measurement results. A program to standardize the methods of reporting, analyzing, comparing, and archiving these data 
is underway between the NASA's GSFC EOS Program Science Office and NIST. Still these preliminary data do provide good examples of the level of agreement of measurements made by the independently calibrated transfer radiometers. Moreover. these data show that the transfer radiometers are capable of validating the radiance scales of integrating spheres to better than the radiometric accuracy specifications of the instruments which employ these spheres as uniform radiance calibration sources.

Figures 1 through 4 show the typical agreement between transfer radiometer measurements at $412 \mathrm{~nm}, 441 \mathrm{~nm}$, and $662 \mathrm{~nm}$ reported during several measurement comparisons. Figure 1 shows agreement at $412 \mathrm{~nm}$ with the average of the transfer radiometer measurements to better than $\pm 2 \%$ for the integrating sphere levels indicated. Figure 2 presents similar data at $441 \mathrm{~nm}$ and shows agreement with the average to better than $\pm 2.5 \%$. Figures 3 and 4 show agreement at $662 \mathrm{~nm}$ with the average to better than $\pm 1.7 \%$. These levels of agreement between the transfer radiometer measurements is remarkable considering that each radiometer is calibrated independently, using standards traceable to NIST in most cases, in each participating institution's metrology laboratory and the only time that transfer radiometer measurements are compared is at the radiometric measurement comparisons.

The level of agreement among the transfer radiometers is commensurate with the estimated relative standard combined uncertainties for the determination of spectral radiance of an unknown source. For the VXR and the SXR, the uncertainty is about $1.5 \% \%^{8,9}$; for the UA VNIR, the uncertainty is about $2.2 \%{ }^{9}$. NRLM assigns a value of $1 \%{ }^{9}$. The $746 / I S I C$ uncertainties are about $5 \%$ at $411 \mathrm{~nm}, 2.5 \%$ at $441 \mathrm{~nm}$, and $1.5 \%$ to $1000 \mathrm{~nm}^{9}$.

Figure 5 presents normalized radiance repeatability measurements obtained using the NIST VXR while viewing integrating spheres at Raytheon SBRS and JPL in August 1996. At each measurement comparison, at least one operating sphere level was designated as a level to be measured at the beginning and close of each measurement day. Interestingly, the data of Figure 5 shows the largest changes in sphere output radiance at $487 \mathrm{~nm}$ and $548 \mathrm{~nm}$.

Figure 6 illustrates the radiance stability measurements from an internal sphere monitor detector and the NIST VXR while viewing the integrating sphere located at the University of Arizona Optical Sciences Center. The internal monitor detector in the UA integrating sphere is a silicon photodiode equipped with a photopic filter. In these measurements, the VXR monitored the integrating sphere at its six wavelengths off-axis while other instruments viewed the sphere on-axis. Only two VXR channels are illustrated; similar results were obtained at the other wavelengths. In Figure 6, the decrease in the sphere output dectected by the UA monitor photodiode is confirmed by the SXR data and is on the order of $0.5 \%$ over 4 hours. The NIST VXR, SXR, and the University of Arizona SWIR radiometers have functioned successfully in a number of measurement comparisons as sphere stability monitors. This capability is particularly important when measuring integrating spheres that are not equipped with filtered monitor photodiode detectors.

The NIST SWIXR, the UA SWIR, the NRLM/JAROS shortwave infrared radiometers, and the GSFC 746/ISIC spectro-radiometer have participated in fewer radiometric measurement comparisons than their visible/near infrared counterparts. Preliminary results from the most recent comparison held at NASA's Ames Research Center in August and September 1999 indicate agreement between the NIST SWIXR, the UA SWIR, and the GSFC $746 /$ SIC at $\pm 2 \%$ at $1646 \mathrm{~nm}$ and $\pm 2.5 \%$ at $2263 \mathrm{~nm}$. It must be emphasized that these results are preliminary, and additional comparisons in the shortwave infrared are needed to fully quantify the level of agreement of these measurements

\section{REFERENCES}

1. B.C. Johnson, J.B. Fowler, and C.L. Cromer, "Volume 1. The SeaWiFS Transfer Radiometer (SXR)," SeaWiFS Postlaunch Technical Report Series, NASA TM-1998-206892, 1998.

2. S.W. Brown, B.C. Johnson, and H.W. Yoon, "Description of a Portable Spectroradiometer to Validate EOS Radiance Scales in the Shortwave Infrared," The Earth Observer 10, 43-48, 1998.

3. S.F. Biggar and P.N. Slater, "Preflight Cross-calibration Radiometers for EOS AM-1 Platform Visible and Near-IR Sources," SPIE 1939, 243-249, 1993.

4. B.L. Markham, J.S. Schafer, F.M. Wood Jr., P.W. Dabney, and J.L. Barker, "Monitoring Large-aperture Spherical Integrating Sources With a Portable Radiometer During Satellite Instrument Calibration," Metrologia 35, 643-648, 1998.

5. F. Sakuma, T. Bret-Dibat, H. Sakate, A. Ono, J. Perbos, J. -M. Martinuzzi, K. Imako, H. Oaku, T. Moriyama, Y. Miyachi, and Y. Tange, "POLDER-OCTS Preflight Cross-calibration Experiment Using Round-robin Radiometers," SPIE 2553, 232-243, 1995. 
6. F. Sakuma, M. Kobayashi, and A. Ono, "ASTER Round-robin Radiometers for the Preflight Cross-calibration of EOS AM-1 Instruments," Proc. IGARSS, 1995-1997, 1994.

7. B.C. Johnson, S.S. Bruce, J.M. Houston, T.R. O'Brian, A. Thompson, S.B. Hooker, and J.L. Mueller, "Volume 37. The Fourth SeaWiFS Intercalibration Round-robin Experiment (SIRREX-4)," SeaWiFS Technical Report Series, NASA TM 104566, 1996.

8. H.W. Yoon, B.C. Johnson, D. Kelch, S. Biggar, and P.R. Spyak, "A $400 \mathrm{~nm}$ to $2500 \mathrm{~nm}$ Absolute Spectral Radiance Comparison using Filter Radiometers," Metrologia 35, 563-568, 1998.

9. B.C. Johnson, F. Sakuma, J.J. Butler, S.F. Biggar, J.W. Cooper, J. Ishida, and K. Suzuki, "Radiometric Measurement Comparison using the Ocean Color Temperature Scanner (OCTS) Visible and Near Infrared Integrating Sphere," J. Res. NIST 102, 627-646, 1997. 


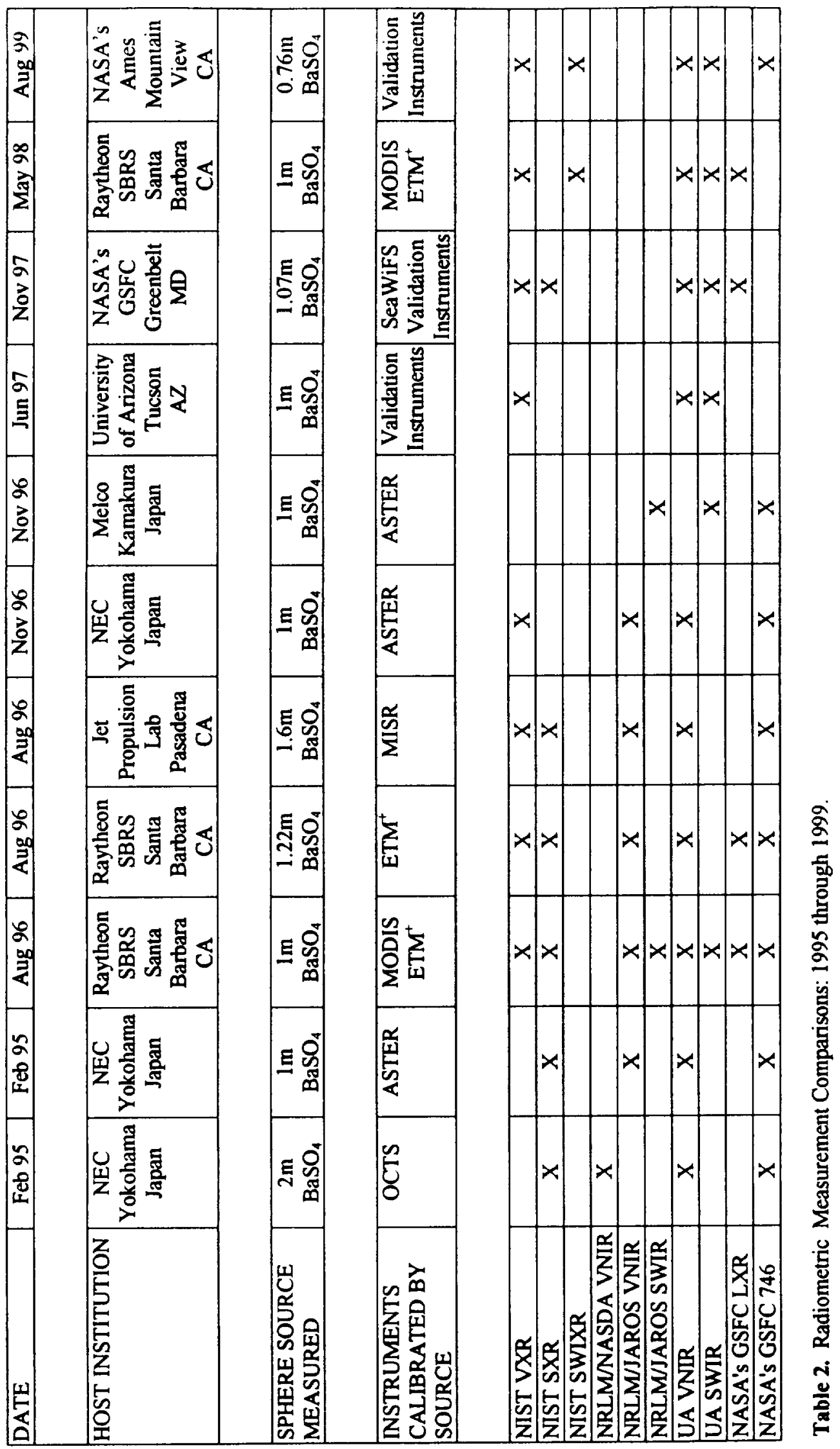



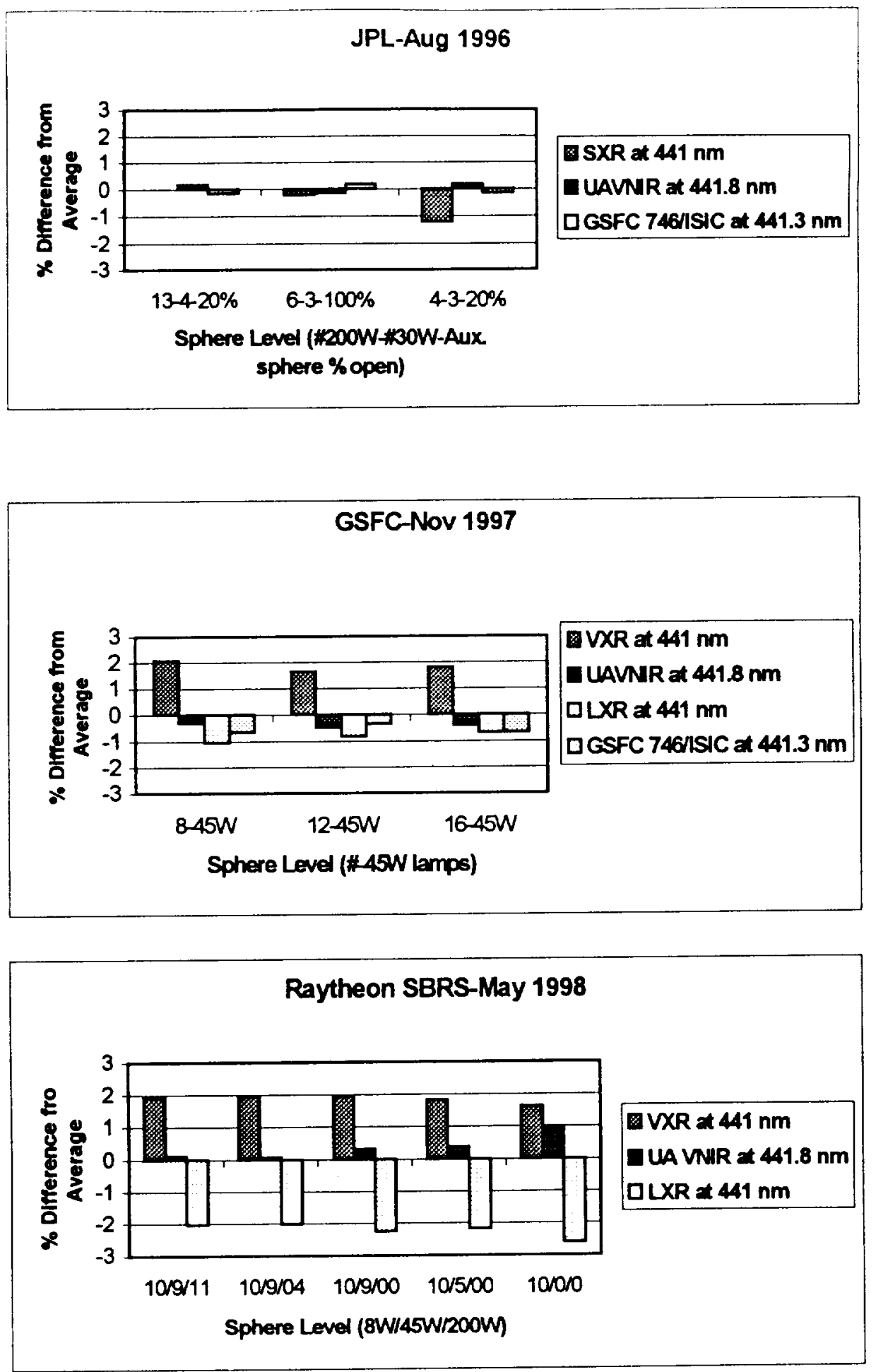

Figure 2. Percent difference of $441 \mathrm{~nm}$ radiance measurements made by the NIST SXR and VXR, the University of Arizona VNIR, the GSFC LXR, and the GSFC 746/ISIC radiometers from the average. These measurements were performed at the Jet Propulsion Laboratory in August 1996, at GSFC in November 1997, and at Raytheon Santa Barbara Remote Sensing in May 1998. The sphere level is indicated in each plot by the number and power of the lamps used and, in the case of the JPL sphere, the percentage of the opening between the principal and auxiliary sphere. 

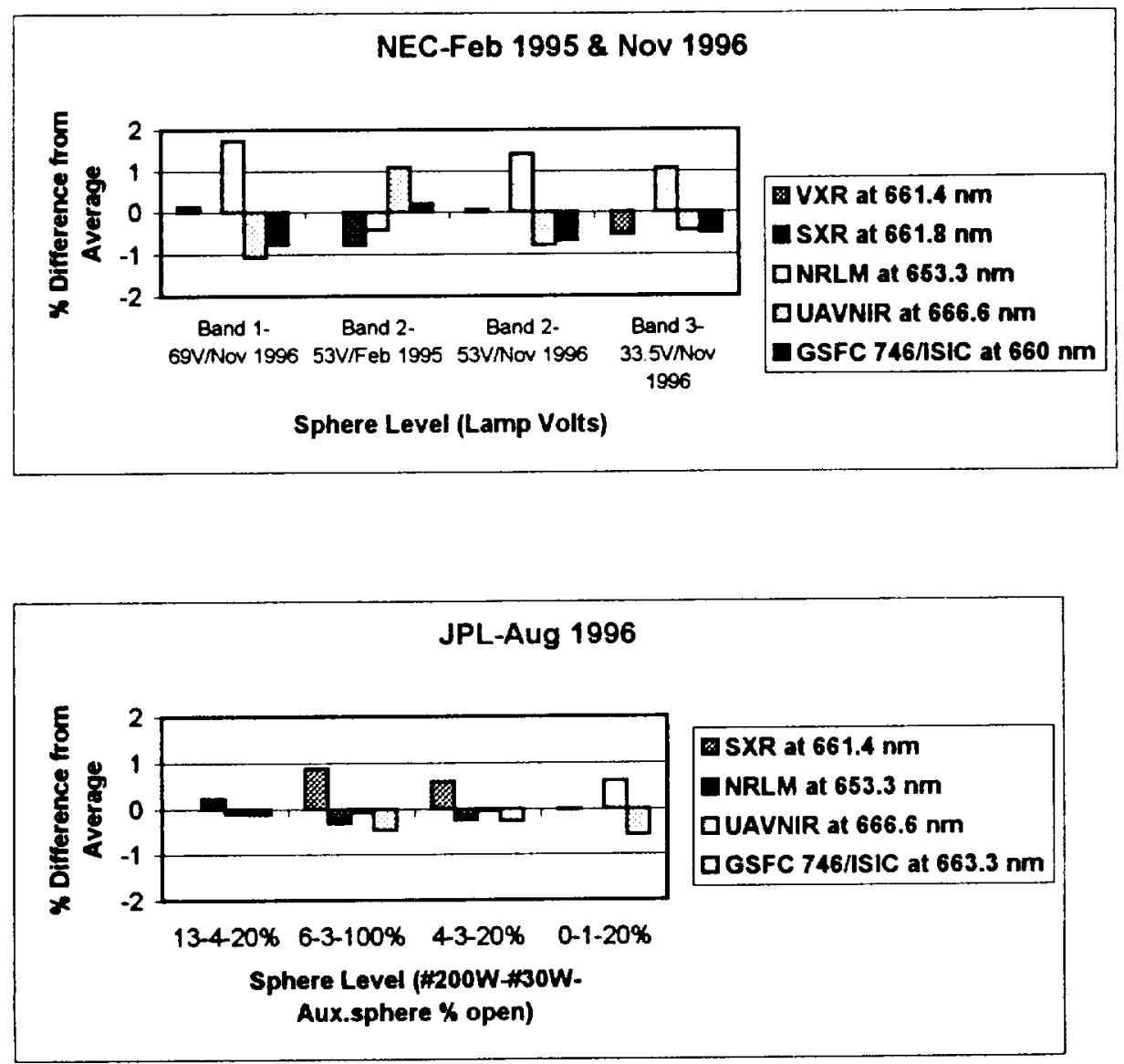

Figure 3. Percent difference of $662 \mathrm{~nm}$ radiance measurements made by the NIST SXR and VXR, the NRLM/JAROS ASTER, the University of Arizona VNIR, and the GSFC 746/ISIC radiometers from the average. These measurements were performed at NEC/Yokohama, Japan in February 1995 and 1996, at the Jet Propulsion Laboratory in August 1996. 

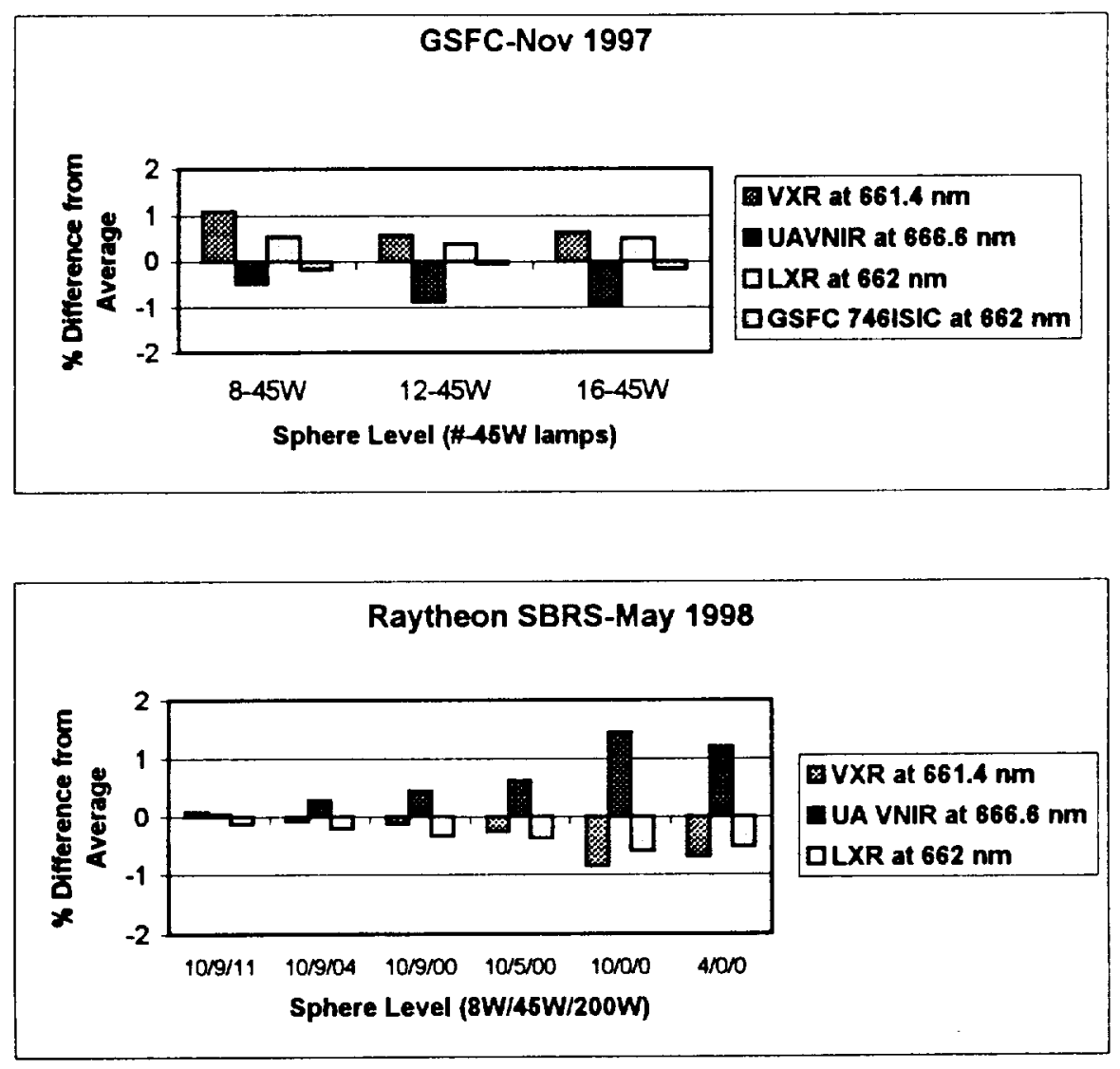

Figure 4. Percent difference of $662 \mathrm{~nm}$ radiance measurements made by the NIST VXR, the University of Arizona VNIR, the GSFC LXR, and the GSFC 746/ISIC radiometers from the average. These measurements were performed at GSFC in November 1997 and at Raytheon Santa Barbara Remote Sensing in May 1998. 

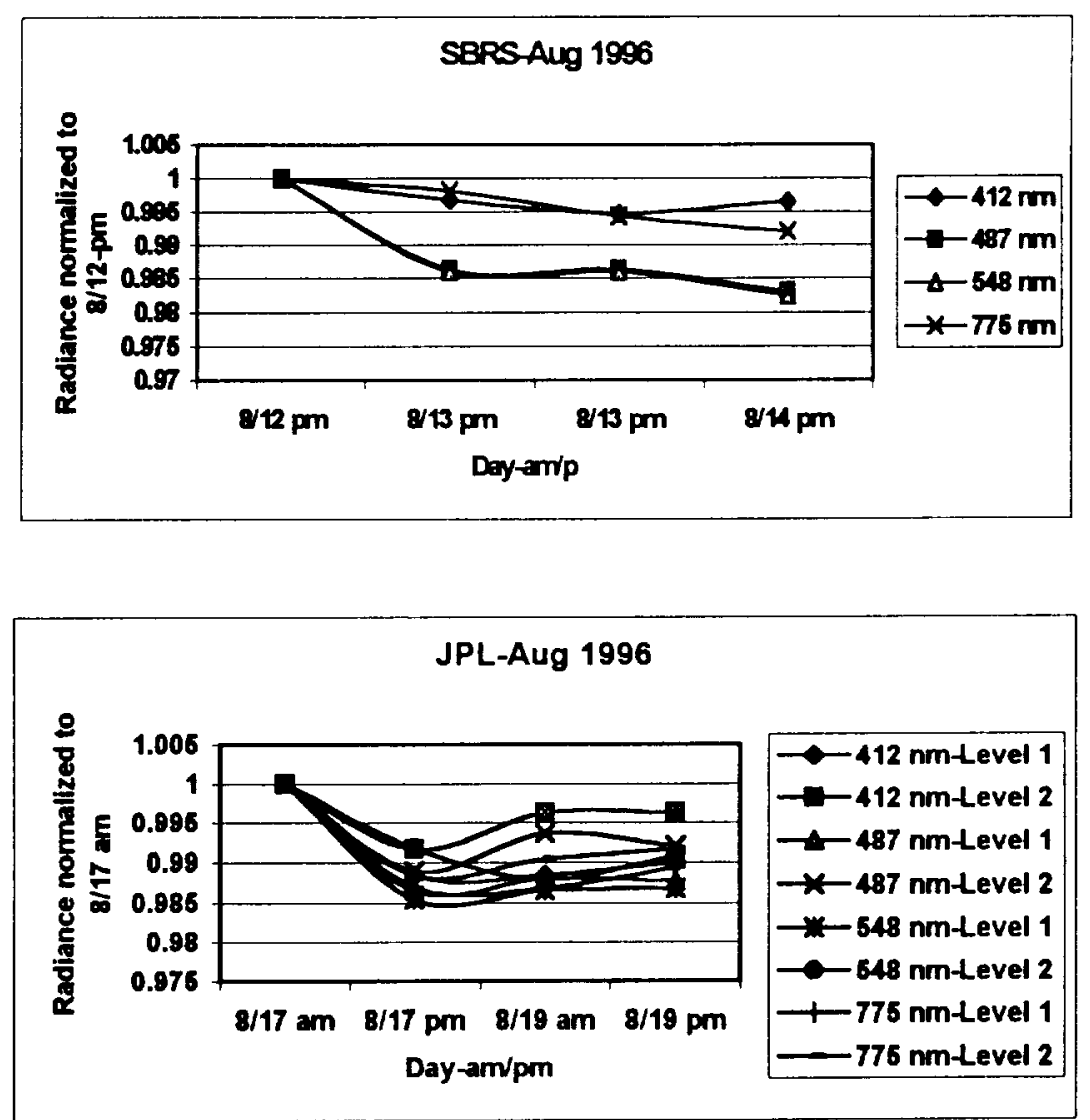

Figure 5. Sphere repeatability measurements using the NIST SXR. These measurements were performed at Raytheon Santa Barbara Remote Sensing and at Jet Propulsion Laboratory in August 1996. For the Raytheon SBRS measurements, the integrating sphere was operated with ten $8 \mathrm{~W}$, nine $45 \mathrm{~W}$, and four $200 \mathrm{~W}$ lamps illuminated. For the JPL measurements, the integrating sphere was operated at Level 1 with four $30 \mathrm{~W}$, thirteen $200 \mathrm{~W}$ illuminated and with the auxiliary sphere aperture $20 \%$ open. The integrating sphere was operated at Level 2 with three $30 \mathrm{~W}$, six $200 \mathrm{~W}$ illuminated and with the auxiliary sphere aperture $100 \%$ open. 

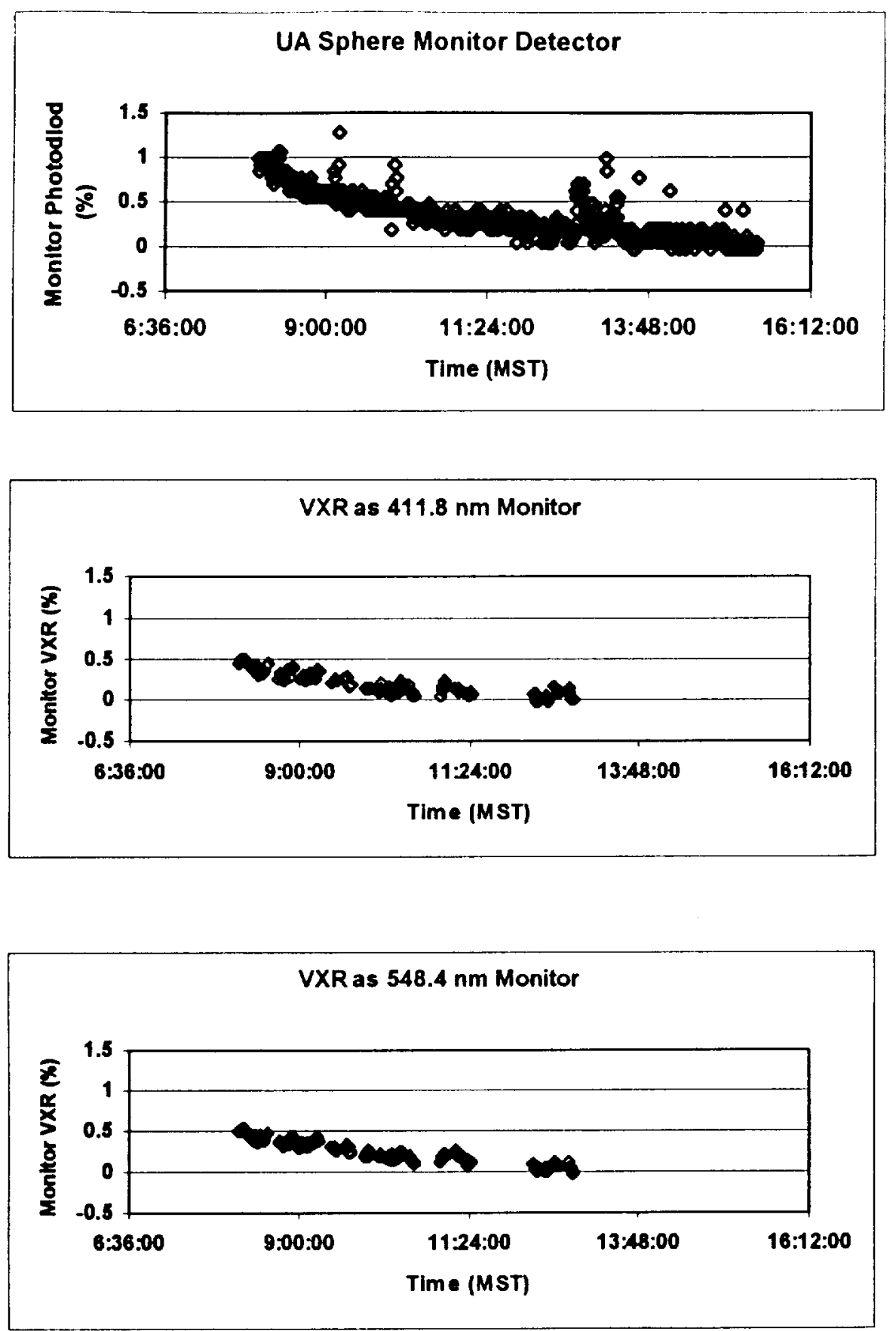

Figure 6. Stability of the UA $1 \mathrm{~m} \mathrm{BaSO}{ }_{4}$ integrating sphere. The top plot shows the percent decrease of the signal measured by the sphere's silicon photodiode monitor detector equipped with a photopic filter. The lower two plots show the percent decrease of the NIST VXR signal at $411.8 \mathrm{~nm}$ and $548.0 \mathrm{~nm}$ when the VXR is used as an off-axis sphere monitor. The time is reported in hr:min:sec Mountain Standard Time (MST). 